\title{
Colloidal Synthesis and Characterization of Tetrapod-Shaped Magnetic Nanocrystals
}

2006

Vol. 6, No. 9

$1966-1972$

\author{
P. Davide Cozzoli,,, Etienne Snoeck, $¥$ Miguel Angel Garcia,§ Cinzia Giannini,," \\ Antonietta Guagliardi," Antonio Cervellino, ${ }^{\perp}$ Fabia Gozzo,", Antonio Hernando, $\$$ \\ Klaus Achterhold, ${ }^{+}$Nelica Ciobanu, ${ }^{+}$Fritz G. Parak, ${ }^{+}$Roberto Cingolani, ${ }^{\dagger}$ and \\ Liberato Manna ${ }^{\dagger}$
}

\begin{abstract}
National Nanotechnology Laboratory of CNR-INFM, Unità di Ricerca IIT, Distretto Tecnologico ISUFI, via per Arnesano km 5, 73100 Lecce, Italy, CEMES-CNRS, 29 rue Jeanne Marvig, B.P. 94347, F-31055 Toulouse Cedex, France, Institute of Applied Magnetism and Department of Materials Physics, UCM, P.O. Box 155, 28230 Las Rozas, Madrid, Spain, Istituto di Cristallografia, Consiglio Nazionale delle Ricerche, via Amendola 122/O, 70126 Bari, Italy, Laboratory for Neutron Scattering, ETH Zurich and PSI Villigen, CH-5232 Villigen PSI, Switzerland, Swiss Light Source, Paul Scherrer Institute, 5232 Villigen PSI, Switzerland, and Physik-Department E17, Technische Universität München, James-Franck-Strasse, D-85747 Garching, Germany
\end{abstract}

Received May 16, 2006; Revised Manuscript Received June 16, 2006

\section{ABSTRACT}

Tetrapod-shaped maghemite nanocrystals are synthesized by manipulating the decomposition of iron pentacarbonyl in a ternary surfactant mixture under mild thermal conditions. Adjustment of the reaction parameters allows for the systematic tuning of both the width and the length of the tetrapod arms, which grow preferentially along the $\langle 111\rangle$ easy axis direction. Such degree of control leads to modulation of the magnetic behavior of the nanocrystals, which evolves systematically as their surface magnetization phase and shape anisotropy are progressively increased.

Colloidal inorganic nanocrystals (NCs) represent model systems for assessing the foundations of dimensionalitydependent physical-chemical laws of nanosized matter. ${ }^{1}$ They also hold promise as versatile building blocks in the fabrication of innovative materials and devices. ${ }^{2}$

To date, wet-chemistry methods have allowed refined control over both the size and shape of NCs for a variety of materials, by delicately balancing thermodynamic parameters (e.g., structure stability and surface energy of the seeds) with kinetically limited growth processes (e.g., active species diffusion, facet-selective surfactant adhesion). ${ }^{3,4}$ Further advances toward structural complexity and versatility have been recently achieved with the preparation of nanocrystal

* To whom all correspondence should be addressed. Phone: +390832 298207. Fax: +390832 298238. E-mail: davide.cozzoli@unile.it.

National Nanotechnology Laboratory of CNR-INFM, Unità di Ricerca IIT, Distretto Tecnologico ISUFI.

CEMES-CNRS

$\S$ Institute of Applied Magnetism and Department of Materials Physics, UCM.

"Istituto di Cristallografia, Consiglio Nazionale delle Ricerche.

${ }^{\perp}$ Laboratory for Neutron Scattering, ETH Zurich and PSI Villigen.

\# Swiss Light Source, Paul Scherrer Institute.

+ Physik-Department E17, Technische Universität München. multipods (such as of $\mathrm{CuCl},{ }^{5} \mathrm{CdSe},{ }^{6} \mathrm{MnO},{ }^{7} \mathrm{PbS},{ }^{8} \mathrm{Au},{ }^{9} \mathrm{Pt},{ }^{10}$ $\mathrm{CdTe},{ }^{11} \mathrm{CdS},{ }^{12}$ and $\mathrm{ZnSe}^{13}$ ), which are particles comprising linear branches connected through a central region. Among such topologies, nanocrystal tetrapods (TPs), in which four arms project out at tetrahedral angles from a branching point, have been already exploited practically in the fabrication of asymmetric nanostructures on surfaces,${ }^{14}$ of nanoscale transistors, ${ }^{15}$ and of solar cells with enhanced electron transport. ${ }^{16}$

Yet, the high yield synthesis of TP-shaped NCs represents an open challenge, as in most cases their formation has been restricted to semiconductor materials exhibiting polymorphism ${ }^{6,11-13}$ or multiple twinned boundaries ${ }^{11}$ that are difficult to control straightforwardly. Single-crystalline TPs have been reported even more rarely for some noble metals. ${ }^{9 b, 10}$ This contribution demonstrates the feasibility of extending the tetrapod shape to iron oxide NCs, for which a rather limited morphological control has been reported so far. ${ }^{17-20}$ Such a class of nontoxic magnetic materials possesses unique properties at the nanoscale, which are currently paving the way to technologically relevant applications such as, for instance, in catalysis, ${ }^{21}$ high-density recording media, ${ }^{22}$ 

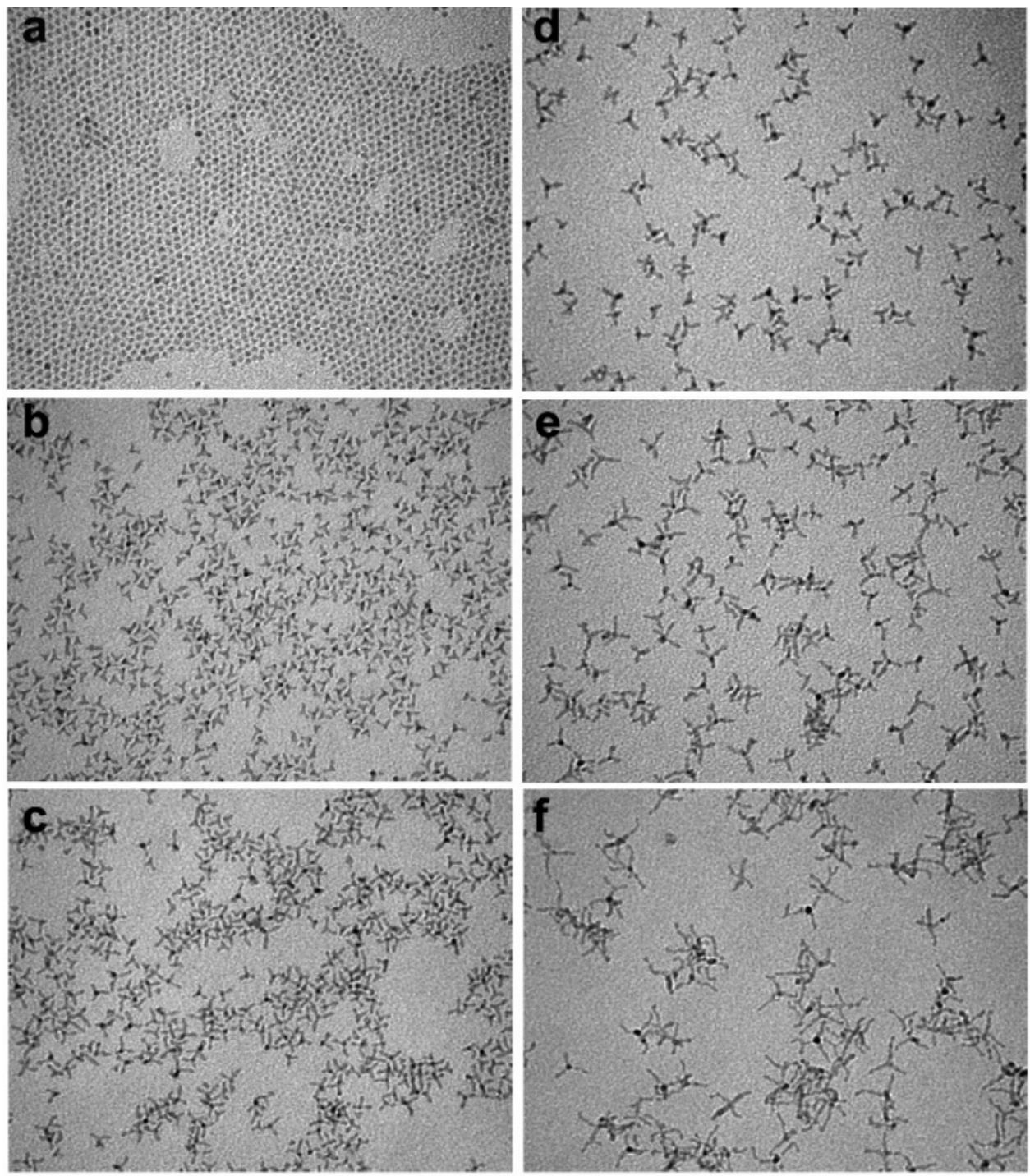

\section{$100 \mathrm{~nm}$}

Figure 1. Low resolution TEM overview of iron oxide nanocrystals synthesized by slowly heating the reaction mixture up to $240{ }^{\circ} \mathrm{C}$. The flask contained $30 \mathrm{~g}$ of ODE, $3 \mathrm{mmol}$ of HDIOL, $1.8 \mathrm{mmol}$ of OLAM, $1.8 \mathrm{mmol}$ of OLAC, and varying amounts of Fe(CO) 5 : (a) 0.8; (b) 1.4; (c) 2; (d) 2.6; (e) 3.2; (f) 4 mmol.

and biomedical diagnostics. ${ }^{23}$ Herein a simple and robust chemical approach is presented, by which it is possible to synthesize colloidal iron oxide TPs in high yields ( $>90 \%)$, with tunable geometric parameters and distinguishable sizedependent magnetic behavior.

In this work, the decomposition of $\mathrm{Fe}(\mathrm{CO})_{5}$ is carried out at $T \leq 240{ }^{\circ} \mathrm{C}$ in octadecene (ODE) in the presence of three different surfactants, namely, oleic acid (OLAC), oleylamine (OLAM), and hexadecan-1,2-diol (HDIOL) at 3:3:5 molar ratio, respectively. After the decomposition of the precursor, oxidation of the resulting product is completed under air at $80{ }^{\circ} \mathrm{C} .{ }^{24}$ The degree of size and shape control achievable by this strategy is demonstrated by the transmission electron microscopy (TEM) overview in Figure 1, which reports the results of various syntheses, all based on the slow heating of a reaction mixture up to a target temperature. Upon increase of the initial $\mathrm{Fe}(\mathrm{CO})_{5}$ concentration in the flask, a deviation from the spherical geometry is observed, as multiarmed particles are formed (with up to a maximum of four branches per particle) and anisotropic growth is progressively enhanced. The tetrapod morphology can be easily inferred from those NCs for which three arms are touching the carbon grid while the fourth arm is pointing upward and is therefore almost parallel to the electron beam. In such cases, a much higher contrast is indeed observed in the central region of the NCs. Remarkably, a continuous tuning of the arm lengths is obtained (from $\sim 3$ to $\sim 30 \mathrm{~nm}$ ) by simply varying the amount of $\mathrm{Fe}(\mathrm{CO})_{5}$ added, while the arm diameter remains almost fixed at around $\sim 3-3.5 \mathrm{~nm}$. Alternatively, the synthesis of TPs can be accomplished by a fast injection of a $\mathrm{Fe}(\mathrm{CO})_{5}$ solution into the same surfactant mixture as above, but already heated at the target temperature. Also in this case the arm length depends on the amount of precursor injected. This technique, on the other hand, yields slightly fatter TPs, with arm diameters as thick as $\sim 5$ nm. ${ }^{24}$ 
To further assess the peculiar three-dimensional morphology of the branched NCs, electron holography experiments were performed in the TEM microscope. Off-axis electron holography is based on the interference between a reference electron wave that has passed through vacuum and one that has been phase shifted following its interaction with an object. ${ }^{25}$ The analysis of the resulting interference pattern, called a hologram, allows the local phase shift of the electron beam to be recovered. The phase shift $\phi$ is sensitive to the electric and magnetic fields in the sample ${ }^{25,26 a}$ and is given by the expression

$$
\phi(x)=C_{\mathrm{E}} \int V_{0}(x, z) \mathrm{d} z-\frac{e}{\hbar} \iint B_{\perp}(x, z) \mathrm{d} x \mathrm{~d} z
$$

where $x$ and $y$ lie in the plane of the sample and $z$ is the incident electron beam direction, $C_{\mathrm{E}}$ is a wavelengthdependent constant $\left(C_{\mathrm{E}}=7.29 \times 10^{6} \mathrm{rad} \mathrm{V}^{-1} \mathrm{~m}^{-1}\right.$ at 200 $\mathrm{kV}), V_{0}$ is the mean electrostatic potential, and $B_{\perp}$ is the component of the magnetic induction (both inside the sample and in the surrounding leakage fields) which is perpendicular to both $x$ and $z$. For the tetrapods of interest here, the electrostatic contribution to $\phi$ is associated solely with the mean inner potential (MIP), i.e., the local composition and thickness of the sample. ${ }^{26}$ Within the high magnetic field of the objective lens (about $1.5 \mathrm{~T}$ ) parallel to the optic axis, the possible magnetization of the tetrapod will be parallel to $z$, and the magnetic contribution to $\phi$ will be therefore zero. If the sample has a uniform composition, $\phi$ becomes

$$
\phi(x, y)=C_{\mathrm{E}} V_{0} t(x, y)
$$

where $V_{0}$ is the MIP for iron oxide and $t(x, y)$ is the thickness of the tetrapod. In panels a and b of Figure 2, a hologram recorded on a single tetrapod and the corresponding phase image are shown, respectively. Assuming that the arms of tetrapod are symmetric, the value of the projected width (i.e., diameter) of the arms in panel a can be taken as the estimate of the arm thickness. Then, the measurement of the phase shift $\phi$ across one arm whose diameter is of $\sim 4 \mathrm{~nm}$ allows determination of a MIP value by using eq 2 . We measured $V_{0}=17 \mathrm{~V}$. Moreover, according to eq $2, \phi$ is proportional to the sample thickness, so that the phase image reflects the three-dimensional (3D) morphology of the particle. Therefore, the phase image can be translated into a surface 3D plot, as displayed in panel c. The maximum phase shift measured at the tetrapod center is about $0.7 \mathrm{rad}$, which corresponds to a particle thickness of $\sim 6 \mathrm{~nm}$ by using the calculated $V_{0}$. This feature can be hence attributed to the fourth arm pointing perpendicular to the surface, which appears as long as the others.

Figure 3 reports a series of high-resolution TEM (HRTEM) micrographs of branched iron oxide NCs. From their fast Fourier transform (FFT) analysis, the NCs are monocrystals, regardless of their size, in the spinel cubic structure of either magnetite $\left(\mathrm{Fe}_{3} \mathrm{O}_{4}\right)$ or maghemite $\left(\gamma-\mathrm{Fe}_{2} \mathrm{O}_{3}\right)$, which cannot be discriminated due to subtle differences between the respective lattice spacings. ${ }^{17-20,26,27}$ The NCs in panels a and
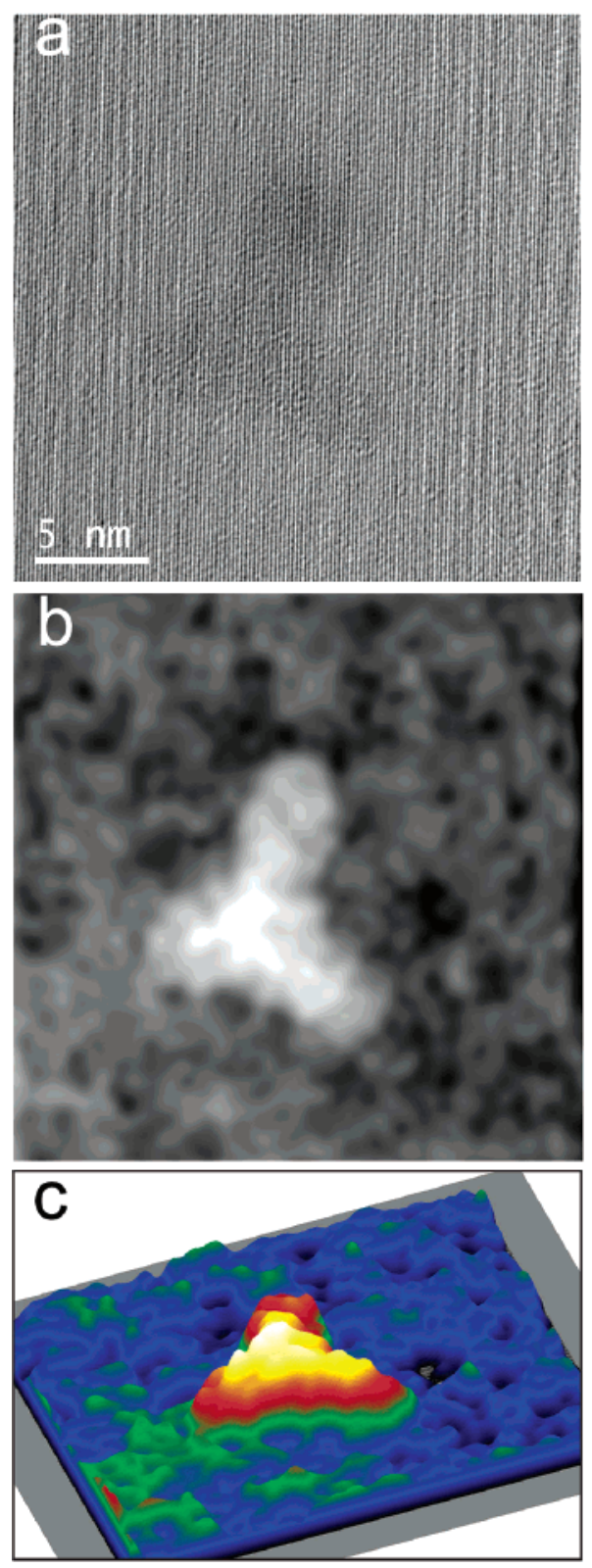

Figure 2. (a) Electron hologram obtained on a tetrapod. (b) Phase shift calculated from the hologram in (a) and from a reference hologram (not shown). (c) 3D surface plot of the phase image in (b).

$\mathrm{b}$ are symmetrical branched particles with a projected 3-fold symmetry and are observed along their 6-fold $\langle 111\rangle$ zone axis. The darker contrast observed in the center of the particle indicates that the fourth arm is pointing perpendicular to the grid plane, parallel to the zone axis. The TPs clearly exhibit $\langle 111\rangle$-oriented branches, although the latter often come less straight in more elongated TPs. Similar deduction on the TP growth mode can be confirmed from the analysis of particles captured in other zone axes (panels c-e). For instance, the TP shown in panel $\mathrm{c}$ is studied along its $\langle 211\rangle$ zone axis and the (111) planes are perpendicular to the axis of the long arm pointing down. Panels $\mathrm{d}$ and e display symmetrical NCs in their $\langle 2 \overline{3} 3\rangle$ and $\langle\overline{2} 11\rangle$ zone axis, respectively. The image contrast at the corresponding branching points suggests that the former is a tetrapod, whereas the latter is more likely a 


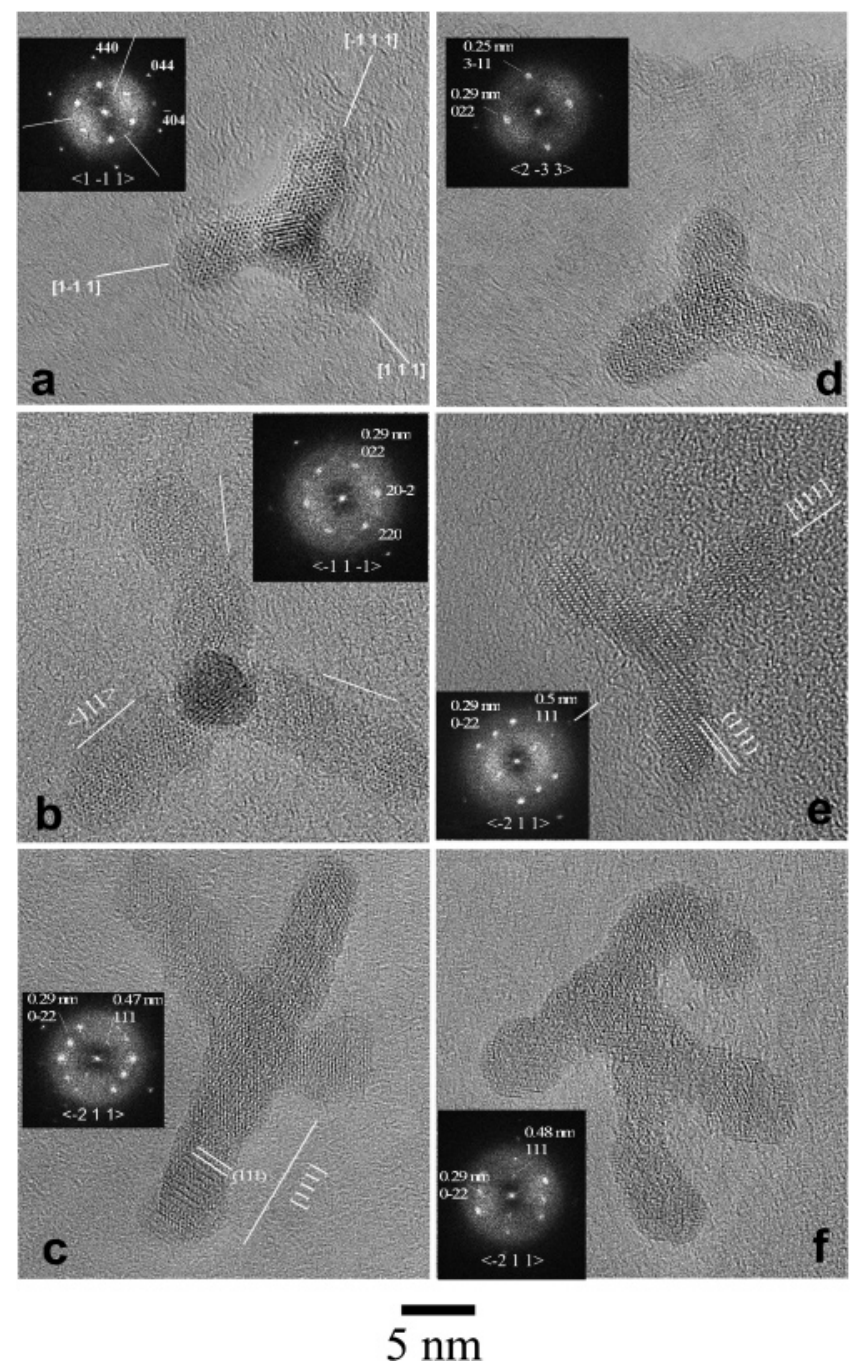

Figure 3. High-resolution TEM images of iron oxide tetrapods in different zone axis. In the insets, the calculated fast Fourier transform of the respective images are reported. The interplanar distances most frequently measured are $0.25,0.29$, and $0.48 \mathrm{~nm}$, which correspond to the (311), (022), (111) planes, respectively, of both magnetite and maghemite.

tripod with $\langle 111\rangle$ elongated branches. Finally, panel f presents an example of a twisted particle, for which, however, no unique growth direction of the arms can be assigned under the observed $\langle\overline{2} 11\rangle$ zone axis.

$\mathrm{X}$-ray diffraction (XRD) experiments and simulations on iron oxide nanocrystal powders confirm the TEM assignments. In Figure 4, the measured powder XRD patterns of iron oxide NCs with varying geometric parameters are reported. Owing to significant line broadening, the patterns can be attributed to the cubic spinel structure of both $\gamma-\mathrm{Fe}_{2} \mathrm{O}_{3}$ and $\mathrm{Fe}_{3} \mathrm{O}_{4}$. It is remarkable that the XRD profiles of TPs exhibit substantial alteration in the relative peak intensity $\left(I_{h k l}\right)$ and widths of several reflections, in comparison with the pattern of roughly spherical iron oxide NCs. A gradual increase in the $I_{440}: I_{311}$ ratio accompanies the transition from the spherical to the TP shape with progressively longer branches. Also, the widths of the (400) and (440) peaks become narrower than those of other reflections and, in addition, the (111) and (220) peak maxima slightly shift to

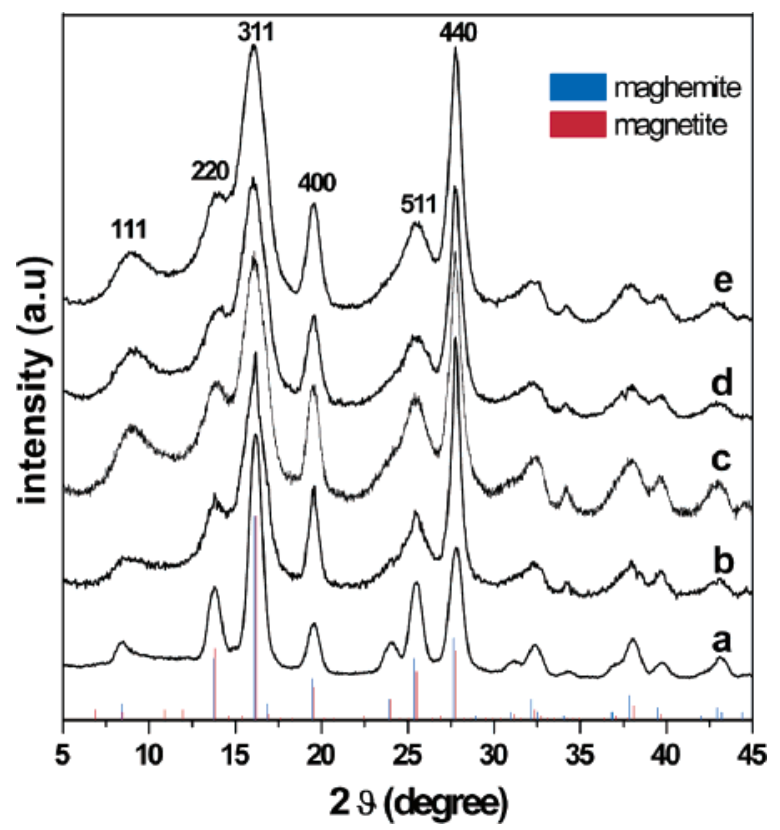

Figure 4. XRD patterns of iron oxide nanocrystals: (a) spheres (corresponding to the sample shown in Figure 2S-b in the Supporting Information); (b-e) tetrapods with increasing dimensions (corresponding to samples in parts b, c, e, and f of Figure 1, respectively).

higher angles. Similar features emerge also in the XRD patterns simulated on atomistic models of TPs with branches elongated in different directions, ${ }^{24}$ which additionally suggest that in our samples TPs with $\langle 111\rangle$ oriented arms could coexist with TPs with $\langle 110\rangle$ oriented arms.

A clearer insight into the crystalline phase composition of the NCs is provided by Mössbauer spectroscopy measurements. In Figure 5 Mössbauer spectra are reported for three representative samples. As shown in the top panel, the differently shaped NCs exhibit the same spectrum at $6 \mathrm{~K}$. The bottom panel reports the temperature-dependent spectra for a TP sample. For the interpretation of the $6 \mathrm{~K}$ data (spectrum h), the parameters obtained for reference materials (namely, bulk magnetite, maghemite, and hematite) have been used. ${ }^{24}$ Only the effective magnetic field (that determines the maximum splitting) and the spectrum area have been varied. The data are explained quite satisfactorily by the $\gamma-\mathrm{Fe}_{2} \mathrm{O}_{3}$ phase. However, if a $10 \%$ contribution from $\mathrm{Fe}_{3} \mathrm{O}_{4}$ is taken into account, the fit improves slightly. It can be concluded that the sample contains mainly $\gamma-\mathrm{Fe}_{2} \mathrm{O}_{3}$ with maximum $10 \%$ of $\mathrm{Fe}_{3} \mathrm{O}_{4}$. This is also consistent with the Mössbauer spectra above $80 \mathrm{~K}$ (spectra $\mathrm{a}-\mathrm{d}$ ) which show a quadrupole splitting in agreement with the literature values for $\gamma-\mathrm{Fe}_{2} \mathrm{O}_{3} \cdot{ }^{27,28}$ Similar conclusions can be drawn from the analysis of other samples.

The effects of size/shape modulation on the magnetic properties of the NCs can be inferred from the data reported in Figure 6 and summarized in Table 1. Figure 6A shows the room-temperature hysteresis loops for a series of samples. These curves show no remanence nor coercivity, regardless of the nanocrystal size and shape, and therefore indicate a superparamagnetic behavior. In all samples, the saturated magnetization $\left(M_{\mathrm{S}}\right)$ values fall below that of bulk $\gamma-\mathrm{Fe}_{2} \mathrm{O}_{3}$ 

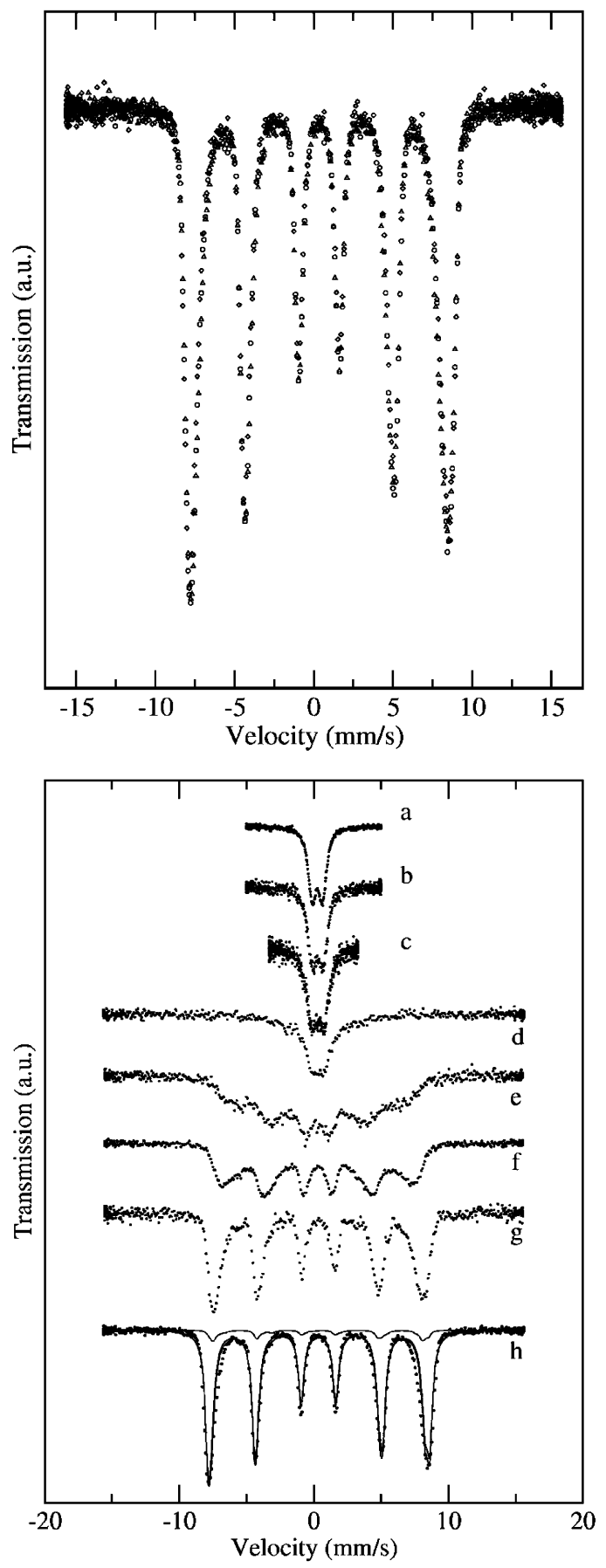

Figure 5. (top) Mössbauer spectra at $6 \mathrm{~K}$ for the three iron oxide samples, corresponding to the nanocrystals shown in Figure 1a (triangle), Figure 1c (diamond), and Figure 1e (circle), respectively. (bottom) Temperature dependence of the Mössbauer spectrum of the sample shown in Figure 1e: (a) $200 \mathrm{~K}$; (b) $160 \mathrm{~K}$; (c) $125 \mathrm{~K}$; (d) $80 \mathrm{~K}$; (e) $50 \mathrm{~K}$; (f) $40 \mathrm{~K}$; (g) $20 \mathrm{~K}$; (h) $6 \mathrm{~K}$. Spectrum h is fitted with $90 \%$ maghemite and $10 \%$ magnetite (thin lines).

$(83.5 \mathrm{emu} / \mathrm{g}$ ). For spherical particles (samples $\mathrm{S} 1-\mathrm{S} 2), M_{\mathrm{S}}$ is reduced as the size decreases, as found previously. ${ }^{29,30}$ Interestingly, in TPs the $M_{\mathrm{S}}$ drops become even more pronounced as the arms elongate (samples TP1-4) and/or their diameter narrows (samples PP1-3). Therefore, the more anisotropically the TPs grow, the more $M_{\mathrm{S}}$ is depressed, despite of the actual increase in the nanocrystal volume. Such
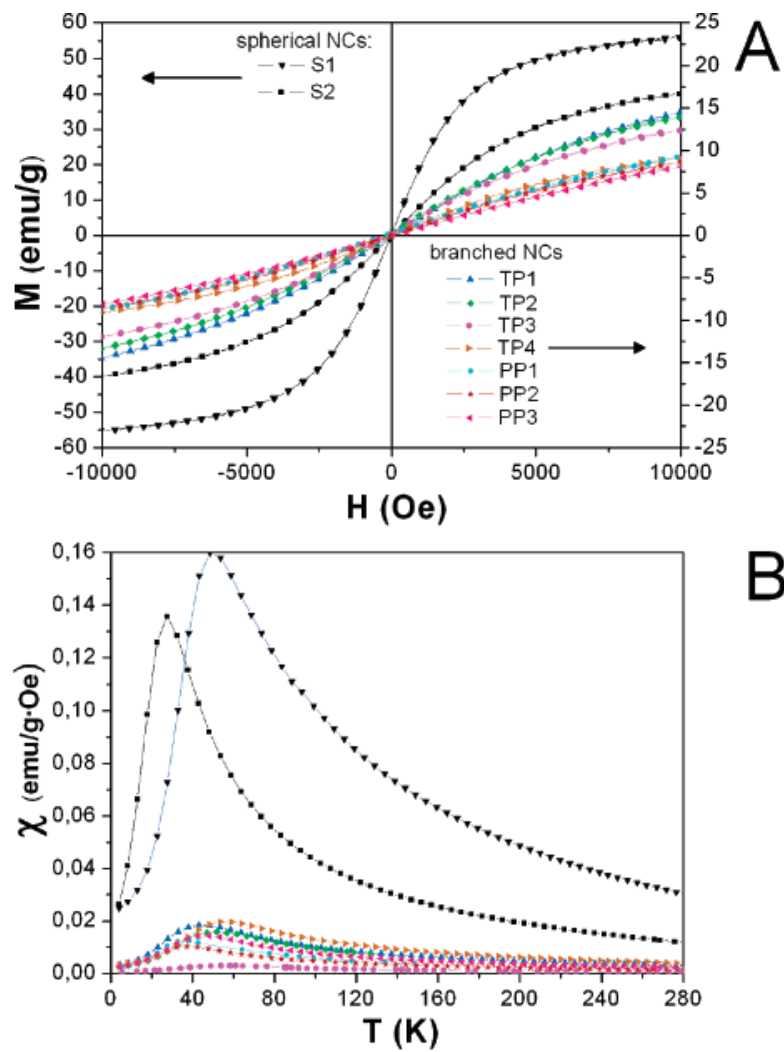

B

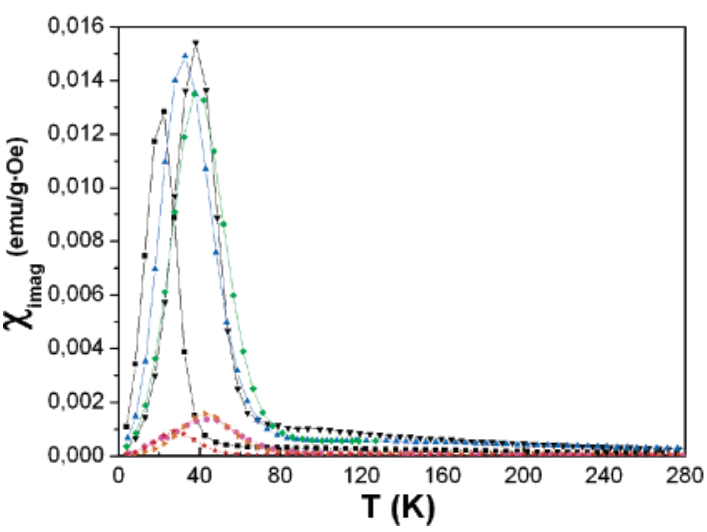

Figure 6. Magnetic measurements for different iron oxide samples (see Table 1 for further details): (A) Hysteresis loops at $300 \mathrm{~K}$ (S1, S2 are referred to the left $y$ axis; other samples are referred to the right $y$ axis); (B) Temperature-dependent ac magnetization susceptibility measured at a field of 20 Oe and a frequency 500 $\mathrm{Hz}$; (C) Imaginary part of the ac susceptibility.

results clearly indicate that the magnetic states of the NCs evolve gradually as a function of both the particle's size and shape.

It is known that in nanosized iron oxide, a phase with 3-4 orders of magnitude lower magnetization is associated with surface atoms which may not be coherently spin-coupled with the inner atoms (due to easy axis anisotropy and/or surface disorder) or which are bound to surfactant molecules. ${ }^{31}$ Our results can be explained well by this concept. The surfaceto-volume ratio $(S / V)$ is higher in a tetrapod than in a sphere, and it increases further as the arms grow longer. It follows that the measured $M_{\mathrm{S}}$ values scale down with increasing the particle $S / V$, as they indeed reflect the proportional increase in the surface phase, which is characterized by a reduced 
Table 1. Magnetic Parameters for the Iron Oxide Nanocrystals Measured in Figure 6

\begin{tabular}{clcc}
\hline $\begin{array}{c}\text { sample } \\
\text { name }\end{array}$ & \multicolumn{1}{c}{ TEM description } & $\begin{array}{c}M_{\mathrm{S}}(1 \mathrm{~T}, 300 \mathrm{~K}) \\
(\mathrm{emu} / \mathrm{g})\end{array}$ & $\begin{array}{c}T_{\mathrm{B}} \\
(\mathrm{K})\end{array}$ \\
\hline S1 & spheres (Figure 1S-b) & 55 & 49 \\
S2 & spheres (Figure 1S-c) & 40.1 & 26 \\
TP1 & tetrapods (Figure 1b) & 14.3 & 43 \\
TP2 & tetrapods (Figure 1c) & 13.8 & 51 \\
TP3 & tetrapods (Figure 1e) & 12.4 & 54 \\
TP & tetrapods (Figure 1f) & 9.2 & 56 \\
PP1 & multipods (Figure 4S-a) $)^{24}$ & 9.06 & 38 \\
PP2 & multipods (Figure 4S-b) $)^{24}$ & 8.4 & 37 \\
PP3 & multipods (Figure 4S-c ${ }^{24}$ & 8.6 & 47
\end{tabular}

magnetization. Further support to this interpretation is provided by the recognition of a clear paramagnetic component (i.e., a linear dependence of $M_{\mathrm{S}} \mathrm{vs} H$ ) which prevents the hysteresis curves from becoming flat at saturation, especially in the samples with the lowest $M_{\mathrm{S}}$ values (see also Table 1).

Figure 6B reports temperature dependence of the ac magnetization susceptibility $\chi$. In all samples, $\chi$ exhibits a maximum in the $40-70 \mathrm{~K}$ region, in correspondence of the average blocking temperature, $T_{\mathrm{B}}$. Accordingly, in Figure $6 \mathrm{C}$ the imaginary part of $\chi$ (i.e., the component out-of-phase with the driving alternate magnetic field), which is related to hysteresis, indeed shows significant values only below this temperature. The $T_{\mathrm{B}}$ values are therefore fully consistent with the superparamagnetic behavior observed at $300 \mathrm{~K}$. It is worth noting that a progressively higher $T_{\mathrm{B}}$ is measured for more anisotropic TPs. The blocking temperature is given by the following expression

$$
T_{\mathrm{B}}=K_{\mathrm{A}} V_{\mathrm{NC}} / 25 K_{\mathrm{B}}
$$

where $V_{\mathrm{NC}}$ is the nanocrystal magnetic volume, $K_{\mathrm{B}}$ is the Boltzmann constant, and the anisotropy constant $K_{\mathrm{A}}$ comprises several contributions. ${ }^{17-20,29-32}$ The $T_{\mathrm{B}}$ value for the TPs scales up with the particle volume as in spheres, ${ }^{28-31}$ although the associated $M_{\mathrm{S}}$ varies in the opposite direction. Such peculiar $T_{\mathrm{B}}$ dependence can be accounted for by considering the effects of the specific morphology on the magnetic states of the NCs. The tetrapods possess arms elongated in the $\langle 111\rangle$ easy axis direction of maghemite, along which the spins will therefore tend to align preferentially. ${ }^{32}$ In a branched shape, turning the magnetization into directions different from that along the easy axes will require higher energy than that available in spheres. ${ }^{18 b, 19,31}$ This fact could lead to increase in $K_{\mathrm{A}}$ and, in turn, in the measured $T_{\mathrm{B}}$. In addition, the TP shape will naturally favor stronger dipolar interactions among the NCs that could contribute to further enhance $T_{\mathrm{B}},{ }^{17-20,29,30}$ thus compensating for the reduced magnetic volume as compared to the case of spheres. In branched NCs with less than four arms, as well as in nanorods, such interactions can be attenuated due to the reduced shape anisotropy, ${ }^{32}$ which can account for the intermediate $T_{\mathrm{B}}$ values measured for these samples (samples PP1-3).
The mechanism through which the $\gamma-\mathrm{Fe}_{2} \mathrm{O}_{3}$ tetrapods are formed is so far unclear. It is known that for the surfactantassisted $\mathrm{Fe}(\mathrm{CO})_{5}$ decomposition the energy barrier for the formation of nanocrystal nuclei is higher than that for their subsequent enlargment, ${ }^{17,29-30}$ as monomer species which contribute to nucleation (i.e., polynuclear iron carbonyl clusters) are distinct from those which participate in the growth process (i.e., Fe-ligand complex molecules). Herein, it has been found that the key strategy to promote tetrapod growth is to combine a ternary surfactant mixture with moderate temperatures. From the chemical point of view, iron oxide is partly formed by thermal decomposition of iron oleate, ${ }^{17,29-30}$ and partly is formed upon air oxidation of the decomposition product of the other iron-ligand species. Under the reaction conditions described in this work, the iron precursor is coordinated strongly by OLAC but more weakly by OLAM and HDIOL, ${ }^{17}$ which would translate into (i) the availability of more reactive $\mathrm{Fe}-\mathrm{OLAM}$ and $\mathrm{Fe}-$ HDIOL complexes sustaining growth only and (ii) easier ligand adsorption-desorption processes on the particle surface, allowing for faster monomer addition to the growing $\mathrm{NCs}$. Increasing the initial amount of $\mathrm{Fe}(\mathrm{CO})_{5}$ injected should therefore lead to greatly enhanced monomer fluxes and promote anisotropic growth. ${ }^{3,4}$ However, reaction temperatures as low as $240{ }^{\circ} \mathrm{C}$ are required to prevent the TPs from being thermally reshaped to more thermodynamically stable spheres. $^{24}$

It is difficult to decouple the specific role played by each surfactant in the present system. Significant deviations (by more than 30-40\%) of the OLAC:OLAM:HDIOL ratio from the optimized ratio of 3:3:5 or the absence of any of the three surfactants suppress tetrapod growth, leading to irregularly shaped or roughly spherical $\mathrm{NCs}^{24}$ This indicates that not only OLAC and OLAM but also HDIOL, which is commonly employed as reductant in many high-temperature nanocrystal syntheses, ${ }^{3}$ should act as shape-directing ligand.

Tetrapod growth does not seem to occur by oriented attachment of initially formed isotropic particles. In fact, branching is observed since the early growth stages and the percentage of branched NCs remains almost constant over time. ${ }^{24}$ Therefore, a facet-selective surfactant adhesion mechanism should be responsible for the shape control of the maghemite NCs under diffusion-limited monomer supply. ${ }^{6,9-13}$ In addition, OLAC appears critical to control the degree of branching, as a decrease in its content indeed promotes the formation of a large fraction of tripods, bipods, and rods. ${ }^{24}$ Overall, the result of triggering tetrapod formation in a complex surfactant mixture at a milder temperature than that used for the synthesis of spherical NCs suggests that this unusual morphology arises from a kinetically controlled growth regime. ${ }^{4}$

In the spinel structure, all eight $\langle 111\rangle$ directions are crystallographically equivalent, so that all (111) facets should have identical growth rates. Hence, in the present case there is no apparent reason why anisotropic growth should develop only along four out of the eight directions, leading to the observed tetrapod morphology. The mechanisms that govern the shape evolution of these NCs are therefore rather 
complicated and cannot be rationalized on the basis of mere crystallographic considerations. Further studies are in progress in our groups to understand the origin of the tetrapod shape in iron oxide NCs.

In conclusion, we have developed a colloidal method for the synthesis of monocrystalline maghemite tetrapods by which we have achieved a fine-tuning of their geometric parameters. This has allowed us to verify shape-dependent magnetic properties that can be expected to extend the technological applications of this class of nontoxic materials. Indeed, tetrapod-shaped NCs are attractive owing to their inherent property to self-align onto a substrate leaving just one arm pointing upward. ${ }^{14-16}$ In addition, the distinguishable magnetic properties are naturally combined with the potential for site-specific NC functionalization (with either molecules or domains of other materials).,33 These features hold promise for the creation of multifunctional nano-objects and of unique hierarchical architectures with intricate spatial arrangements.

Acknowledgment. This work was supported in part by the European project SA-NANO (Contract No. STRP 013698) and the Italian FIRB Contract No. RBLA03ER38.

Supporting Information Available: Details on the tetrapod synthesis; TEM images of iron oxide nanocrystals prepared at higher temperatures and at different surfactant concentration; TEM images of tetrapods at various reaction times; experimental details on TEM, electron holography, XRD, Mössbauer, and magnetic measurements; simulated XRD patterns, Mössbauer spectra of bulk iron oxide materials. This material is available free of charge via the Internet at http://pubs.acs.org.

\section{References}

(1) El-Sayed, M. A. Acc. Chem. Res. 2004, 37, 326.

(2) Cozzoli, P. D.; Manna, L. Nat. Mater. 2005, 4, 801.

(3) Burda, C.; Chen, X.; Narayanan, R.; El-Sayed, M. A. Chem. Rev. 2005, 105, 1025.

(4) Jun, Y.-w.; Lee, J.-H.; Choi, J.-s.; Cheon, J. J. Phys. Chem. B. 2005, 109,14795

(5) Li, Q.; Shao, M.; Yu, G.; Wu, G.; Wu, J.; Li, F.; Qian, Y. J. Mater. Chem. 2003, 13, 424.

(6) (a) Kanaras, A. G.; Sonnichsen, C.; Liu, H.; Alivisatos, A. P. Nano Lett. 2005, 5, 2164. (b) Pang, Q.; Zhao, L.; Cai, Y.; Nguyen, D. P.; Regnault, N.; Wang, N.; Yang, S.; Ge, W.; Ferreira, R.; Bastard, G.; Wang, J. Chem. Mater. 2005, 17, 5263.

(7) (a) Ould-Ely, T.; Prieto-Centurion, D.; Kumar, A.; Guo, W.; Knowles, W. V.; Asokan, S.; Wong, M. S.; Rusakova, I.; Luttge, A.; Whitmire, K. H. Chem. Mater. 2006, 18, 1821. (b) Zitoun, D.; Pinna, N.; Frolet, N.; Belin, C. J. Am. Chem. Soc. 2005, 127, 15034.

(8) (a) Lee, S.-M.; Jun, Y.-w.; Cho, S.-N.; Cheon, J. J. Am. Chem. Soc. 2002, 124, 11244. (b) Zhou, G.; Lu, M.; Xiu, Z.; Wang, S.; Zhang, H.; Zhou, Y.; Wang, S. J. Phys. Chem. B 2006, 110, 6543.

(9) (a) Nehl, C. L.; Liao, H.; Hafner, J. H. Nano Lett. 2006, 6, 683. (b) Chen, S.; Wang, Z. L.; Ballato, J.; Foulger, S. H.; Carroll, D. L. J. Am. Chem. Soc. 2003, 125, 16186. (c) Kuo, C.-H.; Huang, M. H. Langmuir 2005, 21, 2012.
(10) (a) Teng, X.; Yang, H. Nano Lett. 2005, 5, 885. (b) Zhong, X.; Feng, Y., Lieberwirth, I.; Knoll, W. Chem. Mater. 2006, 18, 2468.

(11) Carbone, L.; Kudera, S.; Carlino, E.; Parak, W. P.; Giannini, C.; Cingolani, R.; Manna, L. J. Am. Chem. Soc. 2006, 128, 748.

(12) Jun, Y.-w.; Lee, S.-M.; Kang, N.-J.; Cheon, J. J. Am. Chem. Soc. 2001, 123, 5150.

(13) Cozzoli, P. D.; Manna, L.; Curri, M. L.; Kudera, S.; Giannini, C.; Striccoli, M.; Agostiano, A. Chem. Mater. 2005, 17, 1296.

(14) Liu, H.; Alivisatos, A. P. Nano Lett. 2004, 4, 2397.

(15) Cui, Y.; Banin, U.; Bjork, M. T.; Alivisatos, A. P. Nano Lett. 2005, 5, 1519.

(16) Sun, B.; Marx, E.; Greenham, N. C. Nano Lett. 2003, 3, 961.

(17) (a) Cheon, J.; Kang, N.-J.; Lee, S.-M.; Lee, J.-H.; Yoon, J.-H.; Oh, S. J. J. Am. Chem. Soc. 2004, 126, 1950. (b) Casula, M. F.; Jun, Y.-w.; Zaziski, D. J.; Chan, E. M.; Corrias, A.; Alivisatos, A. P. J. Am. Chem. Soc. 2006, 128, 1675.

(18) (a) Vijaya, R.; Koltypin, Y.; Xu, X. N.; Yeshurun, Y.; Gedanken, A.; Felner, I. J. Appl. Phys. 2001, 89, 6324. (b) Woo, K.; Lee, H. J.; Ahn, J.-P.; Park, Y. S. Adv. Mater. 2003, 15, 1761.

(19) (a) Ngo, A. T.; Pileni, M. P. New J. Phys. 2002, 4, 87.1. (b) Wang, J.; Chen, Q.; Zeng, C.; Hou, B. Adv. Mater. 2004, 16, 137.

(20) Li, S.; Zhang, H.; Wu, J.; Ma, X.; Yang, D. Cryst. Growth Des. 2006, 6, 351 .

(21) (a) He, Y. T.; Traina, S. J. Environ. Sci. Technol. 2005, 39, 4499. (b) Chun, C. L.; Hozalski, R. M.; Arnold, W. A. Environ. Sci. Technol. 2005, 39, 8525.

(22) (a) Speliotis, D. E. J. Magn. Magn. Mater. 1999, 193, 29. (b) Zeng, H.; Li, J.; Liu, J. P.; Wang, Z. L. Sun, S. Nature 2002, 420, 395.

(23) (a) Huh, Y.-M-; Jun, Y.-w; Song, H.-T.; Kim, S.; Choi, J.-S.; Lee, J.-H.; Yoon, S.; Kim, K.-S.; Shin, J.-S.; Suh, J.-S.; Cheon, J. J. Am. Chem. Soc. 2005, 127, 12387. (b) Song, H.-T.; Choi, J.-s.; Huh, Y.M.; Kim, S.; Jun, Y.-w.; Suh, J.-S.; Cheon, J. J. Am. Chem. Soc. 2005, 127, 9992.

(24) See Supporting Information.

(25) (a) Gabor, D. Rev. Mod. Phys. 1956, 28, 260. (b) Tonomura, A. Electron Holography; Springer-Verlag: Berlin, 1993.

(26) (a) Reimer, L. Transmission Electron Microscopy; Springer-Verlag: Berlin, 1997. (b) Lehmann, M.; Lichte, H. Holographic Reconstruction Methods. In Electron Holography; Elsevier: Amsterdam, 1995; pp 69-79.

(27) Tronc, E.; Ezzir, A.; Cherkaoui, R.; ChaneHac, C.; Noguès, M.; Kachkachi, H.; Fiorani, D.; Testa, A. M.; Grenèche, J. M.; Jolivet, J. P J. Magn. Magn. Mater. 2000, 221, 63.

(28) Tueros, M. J.; Baum, L. A.; Borzi, R. A.; Stewart, S. J.; Mercader, R. C.; Marchetti, S. G.; Bengoa, J. F.; Mogni, L. V. Hyperfine Interact. 2003, 148-149, 103.

(29) (a) Park, J.; An, K.; Hwang, Y.; Park, J.-G.; Noh, H.-J.; Kim, J.-Y.; Park, J.-H.; Hwang, N.-M.; Hyeon, T. Nat. Mater. 2004, 3, 891. (b) Park, J.; Lee, E.; Hwang, N.-M.; Kang, M.; Kim, S. C.; Hwang, Y.; Park, J.-G.; Noh, H.-J.; Kim, J.-Y.; Park, J-.H.; Hyeon, T. Angew. Chem., Int. Ed. 2005, 44, 2872.

(30) (a) Sun, S.; Zeng, H, J. Am. Chem. Soc. 2002, 124, 8204. (b) Woo, K.; Hong, J.; Choi, S.; Lee, H.-W.; Ahn, J.-P.; Kim, C. S.; Lee, S. W. Chem. Mater. 2004, 16, 2814. (c) Pinna, N.; Grancharov, S.; Beato, P.; Bonville, P.; Antonietti, N.; Niederberger, M. Chem. Mater. 2005, 17, 3044.

(31) Batlle, X.; Labarta, A. J. Phys. D: Appl. Phys. 2002, 35, R15.

(32) (a) Cordente, N.; Respaud, M.; Senocq, F.; Casanove, M.-J.; Amiens, C.; Chaudret, B. Nano Lett. 2001, 1, 565. (b) Dumestre, F.; Chaudret, B.; Amiens, C.; Respaud, M.; Fejes, P.; Renaud, P.; Zurcher, P. Angew. Chem., Int. Ed. 2003, 42, 5213.

(33) Mokari, T.; Rothenberg, E.; Popov, I.; Costi, R.; Banin, U. Science 2004, 304, 1787

NL061112C 